\title{
The Concurrence of Sexual Violence and Physical Fight Among Adolescent Suicide Ideators and the Risk of Suicide Attempt
}

Xun Li ( $\sim$ li.xunxx@qq.com)

Hunan Children's Hospital

Shi-Ting Xiang

Hunan Children's Hospital

Jie Dong

Hunan Children's Hospital

\section{Research Article}

Keywords: adolescent, physical fight, risk behavior, suicide attempt, suicide ideation, sexual violence

Posted Date: September 20th, 2021

DOI: https://doi.org/10.21203/rs.3.rs-871546/v1

License: (9) This work is licensed under a Creative Commons Attribution 4.0 International License. Read Full License 


\section{Abstract}

Experiences of sexual violence and physical fight had been identified as independent risk factors for suicidal behaviors among adolescents. The question raises whether the concurrence of these two risk factors further increases the risk of suicidal behaviors among ideators, and by how much the risk increases. This study analyzed data from the 2019 National Youth Risk Behavior Surveys. Students that reported had seriously considered attempting suicide in the past year were included $(n=1755)$. The associations between physical fight/sexual violence status and the risk of suicide attempt/plan/injurious attempt were estimated. The concurrence of physical fight and sexual violence substantially increased the relative risks for attempted suicide (relative risk (RR) $=2.01,95 \% \mathrm{Cl}: 1.74,2.31$ ) and injurious suicide attempt ( $\mathrm{RR}=4.03,95 \% \mathrm{Cl}$ : 2.81, 5.78; subgroup analyses: among female, $\mathrm{RR}=3.25,95 \% \mathrm{Cl}$ : $2.09,5.04$; among male, $\mathrm{RR}=6.19,95 \% \mathrm{Cl}$ : $3.3,12.14)$. Among students with concurrent physical fight and sexual violence, males reported more risk behaviors (median=14) than females (median=12) $(P=0.0023)$. The concurrence of physical fight and sexual violence substantially increased the risks for attempted suicide among adolescent suicide ideators. A wide variety of risk behaviors clustered with the concurrence of physical fight and sexual violence, especially among males, which possibly lead to the higher rate of injurious suicide attempt among male students.

\section{Introduction}

Suicide is among the leading causes of death among adolescents worldwide ${ }^{1}$. Globally, suicide was the fourth leading cause of death among the 15-19-year-olds in $2019^{1}$. To take the challenge of reducing the suicide rate among adolescent, better understanding of the adolescents' suicide behaviors is of great importance. It has been noted that only a small subset of those with suicidal ideation will go on to attempt, and even fewer will die by suicide ${ }^{2}$. For example, according to the 2019 National Youth Risk Behavior Surveys (YRBS) from the United States, $18.8 \%$ of the investigated high school students reported having seriously considered suicide, $15.7 \%$ reported having made a suicide plan, $8.9 \%$ reported having attempted suicide, and $2.5 \%$ reported had made a suicide attempt requiring medical treatment ${ }^{3}$. As suicidal ideation alone is not an effective predictor of suicide attempts, identification of risk factors for suicidal behaviors among ideators is important for the development of intervention programmes. Moreover, as past suicide attempt is one of the most consistent risk factors for suicide ${ }^{2}$, and studies had found that a history of suicide attempt was associated with long-standing psychosocial impairment ${ }^{4,5}$, investigations of risk factors and behavior patterns associated with suicide attempts among adolescent suicide ideators could contribute to the health promotions for both youth and adult.

Previous studies had identified a wide range of risk factors associated with the transition from suicide ideators to attempters, ranging from biological to behavior and sociological factors ${ }^{2,6-8}$. However, when considered individually, many of the previously identified risk factors have shown poor prediction of suicidal behavior ${ }^{9-11}$. As individuals who die by suicide usually present with numerous risk factors, rather than a single risk factor in isolation, exploring the effects of combined risk factors might improve our understanding of pathways that may result in suicide attempts and provide novel information for distinguishing suicide attempters from suicide ideators ${ }^{11}$.

Experiences of sexual violence and physical fight had been identified as independent risk factors for suicidal behaviors ${ }^{12-15}$. Their importance in the pathways of adolescent suicidal behaviors is strengthened by the recent study that used the classification tree analysis to identify patterns of risk factors that differentiate adolescent suicide attempters and ideators ${ }^{11}$. The best-performing tree included three classification variables, and lifetime history of rape and past-year physical fight were the first and second classification variable, respectively (the third variable was heroin use). The risk of being attempters was higher among youth characterized by past-year physical fights and heroin use (78\%), youth characterized by past-year physical fights and none heroin use (around $42 \%$ ), and youth who had experienced rape (58\%), compared with youth endorsed none of these three variables (29\%). Although the overall classification accuracy was modest (area under the curve 0.65 ), findings of this study suggested that the association between sexual violence, physical fight, and suicidal behaviors among adolescent worth further investigation.

In our preliminary study, we noticed that there is an overlap between the population experienced sexual violence and the population reported past-year physical fights. According to the interpersonal theory of suicide, for individuals with a suicidal desire, it is possible to acquire the capability for suicide ${ }^{2}$. And both the sexual violence and physical fights could contribute to the capability for suicide. The question raises whether the concurrence of these two risk factors further increases the risk of suicidal behaviors among ideators, and by how much the risk increases. Answers to this question not only helps identify high risk populations, but also improves our understanding of how multiple factors may affect suicide behaviors among ideators. To our knowledge, this question has not yet been explored among adolescents.

With regards to the epidemiology of suicidal behavior, one of the most consistent findings is the sex difference. Worldwide, male deaths by suicide outnumber female deaths, yet female nonlethal suicide attempts outnumber male attempts ${ }^{16}$. As pathways of suicidal behaviors 
might be different among two sexes, we speculate that the effects of sexual violence and physical fights on the risk of suicidal behaviors could be different among two sexes.

Previous studies had shown sexual violence is associated with other health-risk behaviors, like cigarette smoking and marijuana use ${ }^{17}$; and these health-risk behaviors also served as risk factors for suicide behaviors. Similar patterns also observed for physical fights. For example, weapon-carrying is associated with increased risk in physical fight and suicide behaviors ${ }^{18,19}$. According to the problem behavior theory, when adolescents are not provided with a healthy environment, health-risk behaviors and other problem behaviors may cluster together 20. Although the mechanisms of how multiple factors interacted in the suicide pathways could be complex and difficult to conceptualize, based on the problem behavior theory, we propose that individuals exposed to concurrent sexual violence and physical fights would have more health-risk behaviors than individuals with only one of the exposures, and the cluster of health-risk behaviors would further increase the risk of suicidal behaviors.

Drawing from previous study findings and existing theories, we hypothesized that among adolescent suicide ideators: 1) the concurrency of sexual violence and physical fights would increase the risk of suicidal behaviors; 2 ) the effect of concurrent sexual violence and physical fights on suicidal behaviors would be different among sex; 3 ) individuals with the experience of concurrent sexual violence and physical fight would be clustered with more health-risk behaviors, and would result in more severe suicidal behaviors. To test our hypotheses, we used 2019 YRBS data and included students that reported had seriously considered attempting suicide in the past year. According to the responses for the queries on the past-year experience of physical fight and sexual violence, students were categorized into four groups: 1) no physical fight and no sexual violence; 2) sexual violence without physical fight; 3) physical fight without sexual violence; and 4) physical fight and sexual violence. We first described the general characteristics, and the rates of suicide plan, attempted suicide, and injurious suicide attempt according to study groups; and then explored the associations between physical fight/sexual violence status and the risk of suicide attempt/plan/injurious attempt; subgroup analysis according to sex were also conducted; last, we analyzed the distribution of other suicide attempt-associated factors among four groups and compared the cumulative number of risk factors among four groups and different sexes.

\section{Methods}

\section{Data source}

Publicly available data from the 2019 YRBS were analyzed. YRBS is a cross-sectional, school-based survey administered in public and private schools in the U.S. by the Centers for Disease Control. Details on this survey can be found at ${ }^{26}$. One hundred and eighty-one schools were sampled in the 2019 YRBS. Students in grades 9 through 12 were included in the sampling frame. The overall response rate (school response rate multiples the student response rate) was $60.3 \%{ }^{26}$. This study included students reporting past-year seriously considered attempting suicide (Question 26 in the questionnaire). Students seriously considered attempting suicide but had missing data for past-year suicide behaviors (skipped Question 28 for past-year attempted suicide) were excluded.

The national YRBS was approved by the Institutional Review Board (IRB) at the US Centers for Disease Control and Prevention. The participation of students in the YRBS is anonymous and voluntary. Before survey administration, informed consent of parents was obtained according to local parental permission procedures. During survey administration, students completed the self-administered questionnaire during one class period and recorded their responses directly on a computer-scannable booklet ${ }^{26}$. In this study, all YRBS data analyses methods were performed in accordance with the guidelines and regulations provided by the Centers for Disease Control and Prevention.

\section{Measures}

The 2019 YRBS included four queries on suicide ideation and attempt: "Q26. During the past 12 months, did you ever seriously consider attempting suicide? "(answer: Yes/No; variable label: considered suicide); "Q27. During the past 12 months, did you make a plan about how you would attempt suicide? "(answer: Yes/No; variable label: made a suicide plan); "Q28. During the past 12 months, how many times did you actually attempt suicide? " (answer: 0 times/ 1 time/ 2 or 3 times/ 4 or 5 times/ 6 or more times; variable label: attempted suicide); and "Q29. If you attempted suicide during the past 12 months, did any attempt result in an injury, poisoning, or overdose that had to be treated by a doctor or nurse?" (answer: I did not attempt suicide during the past 12 months/Yes/No; variable label: injurious suicide attempt).

The main outcome variable was attempted suicide (Q28). This variable were dichotomized such that responses with one or more suicide attempts were coded as suicide attempt (yes) and responses with zero suicide attempts were coded as suicide attempt (no). The secondary outcome variables were planned suicide (Q27) and injurious suicide attempt (Q29). Injurious suicide attempt were dichotomized such that 
responses of "I did not attempt suicide during the past 12 months" and "No" were coded as injurious suicide attempt (no) and responses of "Yes" was coded as injurious suicide attempt (yes).

Physical fight was measured using the following items: "Q17. During the past 12 months, how many times were you in a physical fight?"; and "Q18. During the past 12 months, how many times were you in a physical fight on school property?". A binary variable was created such that respondents who reported one or more times of physical fight and respondents who reported one or more times of physical fight on school property were coded as past-year physical fight (yes), and respondents reported neither of these experiences were coded as pastyear physical fight (no). Sexual violence was measured using the following items: "Q19. Have you ever been physically forced to have sexual intercourse when you did not want to? "; "Q20. During the past 12 months, how many times did anyone force you to do sexual things that you did not want to do? (Count such things as kissing, touching, or being physically forced to have sexual intercourse.)"; and "Q21. During the past 12 months, how many times did someone you were dating or going out with force you to do sexual things that you did not want to do? (Count such things as kissing, touching, or being physically forced to have sexual intercourse.) ". A binary variable was created such that all respondents with forced sexual intercourse (Q19), all respondents with one or more times of sexual violence (Q20), and all respondents with one or more times of sexual dating violence (Q21) were coded as past-year sexual violence (yes), and respondents reported none of these experiences were coded as past-year sexual violence (no).

According to the status of past-year physical fight and past-year sexual violence, respondents were categorized into four groups: 1) physical fight (no) and sexual violence (no) (physical fight -/ sexual violence -); 2) physical fight (no) and sexual violence (yes) (physical fight -/ sexual violence +); 3) physical fight (yes) and sexual violence (no) (physical fight +/ sexual violence -); and 4) physical fight (yes) and sexual violence (yes)(physical fight + / sexual violence + ).

The associations between 59 risk behaviors investigated in the 2019 YRBS and suicide attempt were screened by Chi-squared test or Fisher's exact test, and 42 risk behaviors were found to be positively associated with the risk of suicide attempt $(P<0.05)$, and these 42 behaviors were included in this study as suicide attempt-associated risk factors (Supplementary Table 1).

\section{Statistical analysis}

Categorical variables were presented as absolute values and percentages. Between-group comparisons for categorical variables were conducted using Chi-squared test or Fisher's exact test, as appropriate. Pairwise comparisons were conducted using Bonferroni correction $\left(a^{\prime}=0.05 /\right.$ number of comparison sets). Log-binomial regression was used to assess the associations between the status of physical fight/sexual violence and suicide behaviors (attempted suicide, planned suicide, and injurious suicide attempt). Relative risk (RR) and $95 \% \mathrm{Cl}$ were calculated and were adjusted for demographic variables that found to be significantly associated with physical fight/sexual violence status. Subgroup analysis for the associations between the status of physical fight/sexual violence and suicide behaviors were conducted according to sex. Attributable risk percent (AR\%) was calculated by (RR-1)/RR $\times 100 \%$. Survey weights were not used because our research questions focused on the associations between physical fight/sexual violence status and suicide attempts among those seriously consider attempting suicide, rather than estimating population prevalence. Missing data was not imputed. All tests were set two-tailed with a type 1 error rate fixed at 5\%. All statistical analyses were performed using SAS 9.3 (SAS Institute, Inc., Cary, NC).

\section{Results}

\section{Study groups and suicide behaviors}

Figure 1 shows the flow chart of the study population. Among the 13677 respondents in the 2019 YRBS, 2633 reported past-year seriously consider attempting suicide. Among these, 538 respondents skipped the query of suicide attempt, 340 respondents skipped the queries for physical fight and/or sexual violence, therefore were excluded from this study. The final analyses included 1755 students. These students were categorized into four groups according to the physical fight/sexual violence status: the (physical fight -/ sexual violence - ) group ( $\mathrm{n}$ $=827,47.12 \%)$; the (physical fight $-/$ sexual violence +$)$ group $(n=360,20.51 \%)$; the (physical fight $+/$ sexual violence -$)$ group $(n=318$, $18.12 \%)$; and the (physical fight + / sexual violence + ) group $(n=250,14.25 \%)$.

Table 1 shows the distribution of demographic characteristics and the rates of past-year suicide attempts among four groups. The distribution of sex, sex identity, and grade were significantly differed among groups $(P<0.05)$. The rates of suicide plan, suicide attempt, and injurious suicide attempt were significantly different among four groups $(P<0.05)$. The (physical fight $+/$ sexual violence +$)$ group had the highest rates of suicide plan (75.5\%), suicide attempt (63.6\%), and injurious suicide attempt (28.4\%). 
Table 1

Demographic characteristics and past-year suicide attempts among students who reported past-year seriously considered attempting suicide in the 2019 YRBS

\begin{tabular}{|c|c|c|c|c|c|c|}
\hline \multirow[t]{2}{*}{ Characteristics } & \multirow[t]{2}{*}{ All a } & \multicolumn{4}{|c|}{ Physical fight/ sexual violence groups } & \multirow[t]{2}{*}{$P$ value } \\
\hline & & $\begin{array}{l}-/- \\
\text { (Group 1) }\end{array}$ & $\begin{array}{l}-/+ \\
\text { (Group 2) }\end{array}$ & $\begin{array}{l}+/- \\
\text { (Group 3) }\end{array}$ & $\begin{array}{l}+/+ \\
\text { (Group 4) }\end{array}$ & \\
\hline Total & 1755 & 827 & 360 & 318 & 250 & \\
\hline \multicolumn{7}{|l|}{ Race } \\
\hline White & $828(48.4)$ & $390(48.3)$ & $178(50.4)$ & $142(46.4)$ & $118(48.2)$ & 0.4722 \\
\hline Black & $216(12.6)$ & $106(13.1)$ & $37(10.5)$ & $42(13.7)$ & $31(12.7)$ & \\
\hline Hispanic & $122(7.1)$ & $68(8.4)$ & $24(6.8)$ & $19(6.2)$ & $11(4.5)$ & \\
\hline Other races/multiple races & $546(31.9)$ & $244(30.2)$ & $114(32.3)$ & $103(33.7)$ & $85(34.7)$ & \\
\hline \multicolumn{7}{|l|}{ Sex } \\
\hline Female & $1129(65.1)$ & $530(64.8)$ & $308(86.3)$ & $123(39.2)$ & $168(68.9)$ & $<0.0001$ \\
\hline Male & $604(34.9)$ & $288(35.2)$ & $49(13.7)$ & $191(60.8)$ & $76(31.1)$ & \\
\hline \multicolumn{7}{|l|}{ Sex identity } \\
\hline Heterosexual & 1085 (63.7) & $530(65.2)$ & $180(52.0)$ & $238(77.5)$ & 137 (57.6) & $<0.0001$ \\
\hline Gay or lesbian & 107 ( 6.3) & $51(6.3)$ & $27(7.8)$ & $13(4.2)$ & $16(6.7)$ & \\
\hline Bisexual & $387(22.7)$ & $168(20.7)$ & $109(31.5)$ & $45(14.7)$ & $65(27.3)$ & \\
\hline Not sure & $125(7.3)$ & $64(7.9)$ & $30(8.7)$ & $11(3.6)$ & $20(8.4)$ & \\
\hline \multicolumn{7}{|l|}{ Grade } \\
\hline 9th grade & 468 (26.9) & $202(24.6)$ & 89 (24.9) & $104(33.2)$ & $73(29.3)$ & $<0.0001$ \\
\hline 10th grade & $489(28.1)$ & $235(28.6)$ & $80(22.3)$ & $91(29.1)$ & $83(33.3)$ & \\
\hline 11 th grade & $411(23.6)$ & $208(25.3)$ & $80(22.3)$ & $72(23.0)$ & $51(20.5)$ & \\
\hline 12 th grade & $373(21.4)$ & $177(21.5)$ & 109 (30.4) & $46(14.7)$ & $41(16.5)$ & \\
\hline Suicide plan & 1143 (65.5) & $503(61.0)$ & 251 (69.9) & $204(64.6)$ & $185(75.5)$ & 0.0001 \\
\hline Suicide attempt & 723 (41.2) & $259(31.3)$ & $172(47.8)$ & $133(41.8)$ & $159(63.6)$ & $<0.0001$ \\
\hline Injurious suicide attempt & $171(11.3)$ & $49(6.7)$ & $36(11.9)$ & $30(10.5)$ & $56(28.4)$ & $<0.0001$ \\
\hline
\end{tabular}

\section{Associations between physical fight/sexual violence status and suicide behaviors}

Table 2 shows the association between physical fight/sexual violence status and suicide behaviors. Compared with the (physical fight $-/$ sexual violence -$)$ group, the other three groups all had significantly increased risk for suicide attempt and injurious suicide attempt $(P$ values $<0.05$ ). The RRs for suicide attempt and injurious suicide attempt among the (physical fight $-/$ sexual violence + ) group and (physical fight + / sexual violence -) group ranged from 1.38 to 1.61 , while remarkably higher RRs were observed among the (physical fight +/ sexual violence + ) group (suicide attempt, $\mathrm{RR}=2.01$; injurious suicide attempt, $\mathrm{RR}=4.03 ; P$ values $<0.05$ ). Weak but significant associations were observed between the (physical fight $-/$ sexual violence + ) group, (physical fight + / sexual violence + ) group, and the risk of suicide plan (RR $=1.1$ and 1.22 , respectively; $P$ values $<0.05)$. 
Table 2

The association between physical fight/ sexual violence status and suicide behaviors

\begin{tabular}{|c|c|c|c|c|c|c|}
\hline Sex & Outcome & Physical fight/ sexual violence & Event \% & $\mathrm{RR}(95 \% \mathrm{Cl})$ & $P$ & $\mathrm{AR} \%$ \\
\hline \multirow[t]{12}{*}{ All } & Suicide attempt & $-/-$ & 31.3 & 1 (Reference) & & 0 \\
\hline & & $-/+$ & 47.8 & $1.48(1.26,1.72)$ & $<0.0001$ & $32.25 \%$ \\
\hline & & $+/-$ & 41.8 & $1.38(1.16,1.64)$ & 0.0003 & $27.73 \%$ \\
\hline & & $+/+$ & 63.6 & $2.01(1.74,2.31)$ & $<0.0001$ & $50.15 \%$ \\
\hline & Suicide plan & $-1-$ & 61 & 1 (Reference) & & 0 \\
\hline & & $-/+$ & 69.9 & $1.1(1,1.2)$ & 0.0419 & $8.94 \%$ \\
\hline & & $+/-$ & 64.6 & $1.1(0.99,1.21)$ & 0.0683 & $8.87 \%$ \\
\hline & & $+/+$ & 75.5 & $1.22(1.11,1.33)$ & $<0.0001$ & $18.02 \%$ \\
\hline & Injurious suicide attempt & $-1-$ & 6.7 & 1 (Reference) & & 0 \\
\hline & & $-/+$ & 11.9 & $1.61(1.04,2.47)$ & 0.0287 & $38.02 \%$ \\
\hline & & $+/-$ & 10.5 & $1.59(1,2.48)$ & 0.0459 & $37.03 \%$ \\
\hline & & $+/+$ & 28.4 & $4.03(2.81,5.78)$ & $<0.0001$ & $75.16 \%$ \\
\hline \multirow[t]{12}{*}{ Female } & Suicide attempt & $-1-$ & 30.9 & 1 (Reference) & & 0 \\
\hline & & $-/+$ & 49 & $1.58(1.33,1.88)$ & $<0.0001$ & $36.81 \%$ \\
\hline & & $+/-$ & 54.5 & $1.75(1.41,2.16)$ & $<0.0001$ & $42.99 \%$ \\
\hline & & $+/+$ & 63.1 & $2(1.67,2.39)$ & $<0.0001$ & $50.06 \%$ \\
\hline & Suicide plan & $-1-$ & 63.8 & 1 (Reference) & & 0 \\
\hline & & $-/+$ & 70.5 & $1.07(0.97,1.18)$ & 0.1487 & $6.92 \%$ \\
\hline & & $+/-$ & 66.4 & $1.05(0.9,1.19)$ & 0.498 & $4.71 \%$ \\
\hline & & $+/+$ & 76 & $1.17(1.05,1.3)$ & 0.0039 & $14.47 \%$ \\
\hline & Injurious suicide attempt & $-1-$ & 7.7 & 1 (Reference) & & 0 \\
\hline & & $-/+$ & 11.5 & $1.48(0.92,2.36)$ & 0.1013 & $32.38 \%$ \\
\hline & & $+/-$ & 14.7 & $1.89(1.04,3.25)$ & 0.0277 & $46.99 \%$ \\
\hline & & $+/+$ & 26.5 & $3.25(2.09,5.04)$ & $<0.0001$ & $69.27 \%$ \\
\hline \multirow[t]{11}{*}{ Male } & Suicide attempt & $-1-$ & 31.9 & 1 (Reference) & & 0 \\
\hline & & $-/+$ & 38.8 & $1.13(0.7,1.67)$ & 0.5917 & $11.16 \%$ \\
\hline & & $+/-$ & 33.5 & $1.06(0.81,1.39)$ & 0.6634 & $5.81 \%$ \\
\hline & & $+/+$ & 64.5 & $2.07(1.63,2.62)$ & $<0.0001$ & $51.74 \%$ \\
\hline & Suicide plan & $-1-$ & 55.4 & 1 (Reference) & & 0 \\
\hline & & $-/+$ & 66.7 & $1.18(0.9,1.46)$ & 0.1777 & $15.10 \%$ \\
\hline & & $+/-$ & 62.6 & $1.16(0.98,1.35)$ & 0.0718 & $13.47 \%$ \\
\hline & & $+/+$ & 74.3 & $1.38(1.15,1.61)$ & 0.0002 & $27.29 \%$ \\
\hline & Injurious suicide attempt & $-1-$ & 5.1 & 1 (Reference) & & 0 \\
\hline & & $-/+$ & 15.8 & $2.35(0.68,6.38)$ & 0.1255 & $57.37 \%$ \\
\hline & & $+/-$ & 7.5 & $1.5(0.7,3.2)$ & 0.2857 & $33.44 \%$ \\
\hline
\end{tabular}

$\mathrm{AR} \%$, attributable risk percent. 


\begin{tabular}{|lllllll|}
\hline Sex Outcome & Physical fight/ sexual violence & Event \% & RR (95\%Cl) & $P$ & AR\% \\
\hline & $+/+$ & 32.8 & $6.19(3.3,12.14)$ & $<0.0001$ & $83.85 \%$ \\
\hline AR\%, attributable risk percent. & & & & & \\
\hline
\end{tabular}

\section{Subgroup analysis according to sex}

In the subgroup analysis (Table 2), the (physical fight +/ sexual violence + ) groups among both sexes showed the highest risk for suicide plan, suicide attempt, and injurious suicide attempt (all $P$ values $<0.05$ ). Different patterns of association were observed among female and male students. Among males, significant associations were only observed in the (physical fight + / sexual violence + ) group. Among females, increased risk of suicide attempt was observed for the (physical fight $-/$ sexual violence + ) group ( $\mathrm{RR}=1.58,95 \% \mathrm{Cl}: 1.33,1.88)$ and the (physical fight +/ sexual violence - ) group ( $R R=1.75,95 \% \mathrm{Cl}: 1.41,2.16)$; and the (physical fight + / sexual violence - ) group also showed an increased risk for injurious suicide attempt $(R R=1.89,95 \% \mathrm{Cl}: 1.04,3.25)$. Notably, strong associations were observed between (physical fight + / sexual violence + ) and injurious suicide attempt among both females (RR $=3.25,95 \% \mathrm{Cl}: 2.09,5.04)$ and males $(R R=6.19$, $95 \% \mathrm{Cl}: 3.3,12.14)$. The AR\% for the injurious suicide attempt were $69.27 \%$ among female and $83.85 \%$ among male.

\section{Suicide attempt-associated factors}

Table 3 shows the distribution of 42 suicide attempt-associated factors among four groups. Compared with the (physical fight $-/$ sexual violence -) group, the rates for most of the investigated risk behaviors in the other three groups were significantly higher. The (physical fight + / sexual violence + ) group had the highest rates for all of the investigated risk behaviors. The risk behavior profiles were more alike between the (physical fight + / sexual violence - ) and (physical fight - / sexual violence + ) groups, except for the rates of weapon carrying, physical dating violence, electronic bullying, initiation of alcohol use ( $\leq 12$ years old), ever ecstasy use, ever sexual intercourse, multiple sex partners, condom use (no) and birth control pill use (no) (between group comparisons $P$ values $<0.05$ ). The cumulative number of risk factors in the (physical fight + / sexual violence - ) group (median $=8$, range: $0-36$ ) and the (physical fight $-/$ sexual violence + ) group (median $=7$, range: 0,26 ) were significantly higher than that in the (physical fight $-/$ sexual violence - ) group (median 4 , range: $0-22$ ). The cumulative number of risk factors were highest among the (physical fight + / sexual violence + ) group (median $=13$, range: $0-40)$. The cumulative number of risk behaviors in each sex was shown in Fig. 2. Among the (physical fight + / sexual violence + ) group, males reported more risk behaviors (median $=14$, range: $2-40$ ) than females (median $=12$, range: $0-33)(P=0.0023)$. The differences in the number of risk behaviors between two sexes in other physical fight/ sexual violence groups were not statistically significant ( $P$ values > 0.05). 
Table 3

Distribution of suicide attempt-associated factors among four physical fight/ sexual violence groups

\begin{tabular}{|c|c|c|c|c|c|c|c|c|c|c|c|}
\hline \multirow[t]{2}{*}{ Risk behavior ${ }^{a}$} & \multirow[t]{2}{*}{ All } & \multicolumn{4}{|c|}{$\begin{array}{l}\text { Physical fight/ sexual violence } \\
\text { groups }\end{array}$} & \multicolumn{6}{|c|}{$P$ values for pair-wise comparisons ${ }^{b}$} \\
\hline & & $\begin{array}{l}-/- \\
\text { (Group } \\
1)\end{array}$ & $\begin{array}{l}-/+ \\
\text { (Group } \\
2 \text { ) }\end{array}$ & $\begin{array}{l}+/- \\
\text { (Group } \\
3 \text { ) }\end{array}$ & $\begin{array}{l}+/+ \\
\text { (Group } \\
\text { 4) }\end{array}$ & $\begin{array}{l}\text { Group } \\
1 \text { vs } 2\end{array}$ & $\begin{array}{l}\text { Group } \\
1 \text { vs } 3\end{array}$ & $\begin{array}{l}\text { Group } \\
1 \text { vs } 4\end{array}$ & $\begin{array}{l}\text { Group } \\
2 \text { vs } 3\end{array}$ & $\begin{array}{l}\text { Group } \\
2 \text { vs } 4\end{array}$ & $\begin{array}{l}\text { Group } \\
3 \text { vs } 4\end{array}$ \\
\hline Total sample size & 1755 & 827 & 360 & 318 & 250 & & & & & & \\
\hline $\begin{array}{l}\text { Seat belt use, } \\
\text { never/rarely }\end{array}$ & $\begin{array}{l}171 \\
(10.2)\end{array}$ & $\begin{array}{l}55( \\
7.0)\end{array}$ & $\begin{array}{l}33( \\
9.6)\end{array}$ & $\begin{array}{l}30( \\
9.8)\end{array}$ & $\begin{array}{l}53 \\
(21.8)\end{array}$ & 0.1315 & 0.1281 & $\begin{array}{l}< \\
0.0001\end{array}$ & 0.9579 & $\stackrel{<}{0.0001}$ & 0.0001 \\
\hline $\begin{array}{l}\text { Riding with a } \\
\text { drinking driver }\end{array}$ & $\begin{array}{l}366 \\
(21.4)\end{array}$ & $\begin{array}{l}131 \\
(16.1)\end{array}$ & $\begin{array}{l}71 \\
(20.5)\end{array}$ & $\begin{array}{l}69 \\
(22.2)\end{array}$ & $\begin{array}{l}95 \\
(39.9)\end{array}$ & 0.0675 & 0.0164 & $\begin{array}{l}< \\
0.0001\end{array}$ & 0.6025 & $\stackrel{<}{0.0001}$ & < 0.0001 \\
\hline Weapon carrying & $\begin{array}{l}322 \\
(19.0)\end{array}$ & $\begin{array}{l}90 \\
(11.0)\end{array}$ & $\begin{array}{l}47 \\
(13.6)\end{array}$ & $\begin{array}{l}94 \\
(30.1)\end{array}$ & $\begin{array}{l}91 \\
(41.0)\end{array}$ & 0.2132 & $\stackrel{<}{0.0001}$ & $\stackrel{<}{0.0001}$ & $\stackrel{<}{0.0001}$ & $\stackrel{<}{0.0001}$ & 0.0093 \\
\hline $\begin{array}{l}\text { Weapon carrying } \\
\text { at school }\end{array}$ & $\begin{array}{l}72( \\
4.3)\end{array}$ & $\begin{array}{l}15( \\
1.9)\end{array}$ & $\begin{array}{l}11( \\
3.2)\end{array}$ & $\begin{array}{l}10( \\
3.2)\end{array}$ & $\begin{array}{l}36 \\
(15.3)\end{array}$ & 0.1837 & 0.1734 & $\begin{array}{l}< \\
0.0001\end{array}$ & 0.9503 & $\stackrel{<}{0.0001}$ & $\begin{array}{l}< \\
0.0001\end{array}$ \\
\hline $\begin{array}{l}\text { Gun carrying past } \\
12 \text { mos }\end{array}$ & $\begin{array}{l}75( \\
5.1)\end{array}$ & $\begin{array}{l}10( \\
1.4)\end{array}$ & $\begin{array}{l}9( \\
3.1)\end{array}$ & $\begin{array}{l}22( \\
7.9)\end{array}$ & $\begin{array}{l}34 \\
(18.3)\end{array}$ & 0.0772 & $\stackrel{<}{0.0001}$ & $\begin{array}{l}< \\
0.0001\end{array}$ & 0.0098 & $\stackrel{<}{0.0001}$ & 0.0008 \\
\hline $\begin{array}{l}\text { Safety concerns at } \\
\text { school }\end{array}$ & $\begin{array}{l}295 \\
(16.8)\end{array}$ & $\begin{array}{l}78( \\
9.4)\end{array}$ & $\begin{array}{l}76 \\
(21.2)\end{array}$ & $\begin{array}{l}52 \\
(16.4)\end{array}$ & $\begin{array}{l}89 \\
(35.7)\end{array}$ & $\begin{array}{l}< \\
0.0001\end{array}$ & 0.0009 & $\stackrel{<}{0.0001}$ & 0.1101 & 0.0001 & $\begin{array}{l}< \\
0.0001\end{array}$ \\
\hline $\begin{array}{l}\text { Threatened at } \\
\text { school }\end{array}$ & $\begin{array}{l}270 \\
(15.5)\end{array}$ & $\begin{array}{l}65( \\
7.9)\end{array}$ & $\begin{array}{l}52 \\
(14.5)\end{array}$ & $\begin{array}{l}68 \\
(21.6)\end{array}$ & $\begin{array}{l}85 \\
(34.3)\end{array}$ & 0.0005 & $\begin{array}{l}< \\
0.0001\end{array}$ & $\begin{array}{l}< \\
0.0001\end{array}$ & 0.0169 & $\stackrel{<}{0.0001}$ & 0.0008 \\
\hline $\begin{array}{l}\text { Physical dating } \\
\text { violence }\end{array}$ & $\begin{array}{l}209 \\
(12.3)\end{array}$ & $\begin{array}{l}31( \\
3.9)\end{array}$ & $\begin{array}{l}71 \\
(20.5)\end{array}$ & $\begin{array}{l}25( \\
8.2)\end{array}$ & $\begin{array}{l}82 \\
(34.0)\end{array}$ & $\begin{array}{l}< \\
0.0001\end{array}$ & 0.0033 & $\begin{array}{l}< \\
0.0001\end{array}$ & $\begin{array}{l}< \\
0.0001\end{array}$ & 0.0002 & < 0.0001 \\
\hline Bullying at school & $\begin{array}{l}705 \\
(40.6)\end{array}$ & $\begin{array}{l}245 \\
(29.8)\end{array}$ & $\begin{array}{l}180 \\
(50.6)\end{array}$ & $\begin{array}{l}133 \\
(42.1)\end{array}$ & $\begin{array}{l}147 \\
(60.5)\end{array}$ & $\stackrel{<}{0.0001}$ & 0.0001 & $\stackrel{<}{0.0001}$ & 0.0280 & 0.0165 & < 0.0001 \\
\hline Electronic bullying & $\begin{array}{l}581 \\
(33.3)\end{array}$ & $\begin{array}{l}183 \\
(22.2)\end{array}$ & $\begin{array}{l}157 \\
(43.9)\end{array}$ & $\begin{array}{l}107 \\
(33.6)\end{array}$ & $\begin{array}{l}134 \\
(55.4)\end{array}$ & $\begin{array}{l}< \\
0.0001\end{array}$ & 0.0001 & $\begin{array}{l}< \\
0.0001\end{array}$ & 0.0066 & 0.0056 & $\stackrel{<}{0.0001}$ \\
\hline Ever cigarette use & $\begin{array}{l}545 \\
(36.0)\end{array}$ & $\begin{array}{l}177 \\
(24.3)\end{array}$ & $\begin{array}{l}124 \\
(40.3)\end{array}$ & $\begin{array}{l}117 \\
(42.4)\end{array}$ & $\begin{array}{l}127 \\
(63.8)\end{array}$ & $\begin{array}{l}< \\
0.0001\end{array}$ & $\begin{array}{l}< \\
0.0001\end{array}$ & $\begin{array}{l}< \\
0.0001\end{array}$ & 0.6014 & $\begin{array}{l}< \\
0.0001\end{array}$ & $\begin{array}{l}< \\
0.0001\end{array}$ \\
\hline $\begin{array}{l}\text { Initiation of } \\
\text { cigarette smoking, } \\
\leq 12 \text { years old }\end{array}$ & $\begin{array}{l}214 \\
(12.6)\end{array}$ & $\begin{array}{l}52( \\
6.4)\end{array}$ & $\begin{array}{l}45 \\
(13.0)\end{array}$ & $\begin{array}{l}48 \\
(15.7)\end{array}$ & $\begin{array}{l}69 \\
(30.4)\end{array}$ & 0.0002 & $\begin{array}{l}< \\
0.0001\end{array}$ & $\begin{array}{l}< \\
0.0001\end{array}$ & 0.3276 & $\begin{array}{l}< \\
0.0001\end{array}$ & 0.0001 \\
\hline $\begin{array}{l}\text { Current cigarette } \\
\text { use }\end{array}$ & $\begin{array}{l}168 \\
(11.2)\end{array}$ & $\begin{array}{l}44( \\
6.1)\end{array}$ & $\begin{array}{l}39 \\
(12.8)\end{array}$ & $\begin{array}{l}28 \\
(10.0)\end{array}$ & $\begin{array}{l}57 \\
(28.8)\end{array}$ & 0.0003 & 0.0295 & $\begin{array}{l}< \\
0.0001\end{array}$ & 0.2974 & $\stackrel{<}{0.0001}$ & $\stackrel{<}{0.0001}$ \\
\hline $\begin{array}{l}\text { Smoked > } 10 \\
\text { cigarettes }\end{array}$ & $\begin{array}{l}16( \\
1.0)\end{array}$ & $\begin{array}{l}2( \\
0.2)\end{array}$ & $\begin{array}{l}2( \\
0.6)\end{array}$ & $\begin{array}{l}1( \\
0.3)\end{array}$ & $\begin{array}{l}11( \\
4.9)\end{array}$ & 0.3772 & 0.8139 & $\begin{array}{l}< \\
0.0001\end{array}$ & 0.6373 & 0.0009 & 0.0006 \\
\hline $\begin{array}{l}\text { Electronic vapor } \\
\text { product use }\end{array}$ & $\begin{array}{l}1121 \\
(65.3)\end{array}$ & $\begin{array}{l}448 \\
(54.8)\end{array}$ & $\begin{array}{l}259 \\
(73.8)\end{array}$ & $\begin{array}{l}221 \\
(70.8)\end{array}$ & $\begin{array}{l}193 \\
(81.4)\end{array}$ & $\begin{array}{l}< \\
0.0001\end{array}$ & $\begin{array}{l}< \\
0.0001\end{array}$ & $\begin{array}{l}< \\
0.0001\end{array}$ & 0.3954 & 0.0310 & 0.0043 \\
\hline $\begin{array}{l}\text { Current electronic } \\
\text { vapor product use }\end{array}$ & $\begin{array}{l}725 \\
(44.2)\end{array}$ & $\begin{array}{l}261 \\
(33.2)\end{array}$ & $\begin{array}{l}172 \\
(51.0)\end{array}$ & $\begin{array}{l}149 \\
(50.3)\end{array}$ & $\begin{array}{l}143 \\
(63.8)\end{array}$ & $\begin{array}{l}< \\
0.0001\end{array}$ & $\begin{array}{l}< \\
0.0001\end{array}$ & $\begin{array}{l}< \\
0.0001\end{array}$ & 0.8603 & 0.0028 & 0.0021 \\
\hline $\begin{array}{l}\text { Current smokeless } \\
\text { tobacco use }\end{array}$ & $\begin{array}{l}75( \\
4.4)\end{array}$ & $\begin{array}{l}10( \\
1.2)\end{array}$ & $\begin{array}{l}10( \\
2.8)\end{array}$ & $\begin{array}{l}21( \\
6.9)\end{array}$ & $\begin{array}{l}34 \\
(14.3)\end{array}$ & 0.0522 & $\stackrel{<}{0.0001}$ & $\begin{array}{l}< \\
0.0001\end{array}$ & 0.0155 & $\stackrel{<}{0.0001}$ & 0.0042 \\
\hline Current cigar use & $\begin{array}{l}138( \\
8.1)\end{array}$ & $\begin{array}{l}27( \\
3.3)\end{array}$ & $\begin{array}{l}26( \\
7.4)\end{array}$ & $\begin{array}{l}37 \\
(12.2)\end{array}$ & $\begin{array}{l}48 \\
(20.4)\end{array}$ & 0.0024 & $\begin{array}{l}< \\
0.0001\end{array}$ & $\begin{array}{l}< \\
0.0001\end{array}$ & 0.0379 & $\begin{array}{l}< \\
0.0001\end{array}$ & 0.0096 \\
\hline $\begin{array}{l}\text { Initiation of } \\
\text { alcohol use, } \leq 12 \\
\text { years old }\end{array}$ & $\begin{array}{l}426 \\
(24.9)\end{array}$ & $\begin{array}{l}119 \\
(14.7)\end{array}$ & $\begin{array}{l}85 \\
(24.0)\end{array}$ & $\begin{array}{l}105 \\
(34.2)\end{array}$ & $\begin{array}{l}117 \\
(48.3)\end{array}$ & 0.0001 & $\begin{array}{l}< \\
0.0001\end{array}$ & $\begin{array}{l}< \\
0.0001\end{array}$ & 0.0039 & $\begin{array}{l}< \\
0.0001\end{array}$ & 0.0008 \\
\hline
\end{tabular}

a. Risk factors that significantly associated with past- year suicide attempt. The full description for the question and response classification were presented in Supplementary Table 1.

b. Bold values were statistically significant after Bonferroni correction $\left(a^{\prime}=0.05 / 6=0.0083\right)$. 


\begin{tabular}{|c|c|c|c|c|c|c|c|c|c|c|c|}
\hline \multirow[t]{2}{*}{ Risk behavior a } & \multirow[t]{2}{*}{ All } & \multicolumn{4}{|c|}{$\begin{array}{l}\text { Physical fight/ sexual violence } \\
\text { groups }\end{array}$} & \multicolumn{6}{|c|}{$P$ values for pair-wise comparisons ${ }^{b}$} \\
\hline & & $\begin{array}{l}-/- \\
\text { (Group } \\
\text { 1) }\end{array}$ & $\begin{array}{l}-/+ \\
\text { (Group } \\
2 \text { ) }\end{array}$ & $\begin{array}{l}+/- \\
\text { (Group } \\
3 \text { ) }\end{array}$ & $\begin{array}{l}+/+ \\
\text { (Group } \\
4 \text { ) }\end{array}$ & $\begin{array}{l}\text { Group } \\
1 \text { vs } 2\end{array}$ & $\begin{array}{l}\text { Group } \\
1 \text { vs } 3\end{array}$ & $\begin{array}{l}\text { Group } \\
1 \text { vs } 4\end{array}$ & $\begin{array}{l}\text { Group } \\
2 \text { vs } 3\end{array}$ & $\begin{array}{l}\text { Group } \\
2 \text { vs } 4\end{array}$ & $\begin{array}{l}\text { Group } \\
3 \text { vs } 4\end{array}$ \\
\hline $\begin{array}{l}\text { Current alcohol } \\
\text { use }\end{array}$ & $\begin{array}{l}657 \\
(40.8)\end{array}$ & $\begin{array}{l}222 \\
(28.6)\end{array}$ & $\begin{array}{l}157 \\
(48.0)\end{array}$ & $\begin{array}{l}132 \\
(45.7)\end{array}$ & $\begin{array}{l}146 \\
(66.1)\end{array}$ & $\hat{0} 0001$ & $\hat{0} 0001$ & $\dot{0} 0001$ & 0.5618 & $<.0001$ & $\hat{0} .0001$ \\
\hline $\begin{array}{l}\text { Current binge } \\
\text { drinking }\end{array}$ & $\begin{array}{l}300 \\
(18.7)\end{array}$ & $\begin{array}{l}96 \\
(12.2)\end{array}$ & $\begin{array}{l}69 \\
(21.5)\end{array}$ & $\begin{array}{l}58 \\
(20.1)\end{array}$ & $\begin{array}{l}77 \\
(37.4)\end{array}$ & 0.0001 & 0.0010 & $\dot{\delta} 0001$ & 0.6649 & 0.0001 & $\overleftarrow{0.0001}$ \\
\hline $\begin{array}{l}\text { Largest number of } \\
\text { drinks, } \geq 10 \text { drinks }\end{array}$ & $\begin{array}{l}64( \\
4.8)\end{array}$ & $\begin{array}{l}14( \\
2.1)\end{array}$ & $\begin{array}{l}13( \\
5.0)\end{array}$ & $\begin{array}{l}11( \\
4.6)\end{array}$ & $\begin{array}{l}26 \\
(15.4)\end{array}$ & 0.0215 & 0.0478 & $\hat{0} .0001$ & 0.8426 & 0.0002 & 0.0002 \\
\hline $\begin{array}{l}\text { Ever marijuana } \\
\text { use }\end{array}$ & $\begin{array}{l}807 \\
(54.1)\end{array}$ & $\begin{array}{l}311 \\
(43.3)\end{array}$ & $\begin{array}{l}185 \\
(60.1)\end{array}$ & $\begin{array}{l}162 \\
(59.8)\end{array}$ & $\begin{array}{l}149 \\
(76.8)\end{array}$ & $\hat{0} .0001$ & $<.0001$ & $\hat{0} .0001$ & 0.9441 & 0.0001 & 0.0001 \\
\hline $\begin{array}{l}\text { Initiation of } \\
\text { marijuana use, } \leq \\
12 \text { years old }\end{array}$ & $\begin{array}{l}169( \\
9.8)\end{array}$ & $\begin{array}{l}40( \\
4.9)\end{array}$ & $\begin{array}{l}29( \\
8.2)\end{array}$ & $\begin{array}{l}41 \\
(13.4)\end{array}$ & $\begin{array}{l}59 \\
(24.4)\end{array}$ & 0.0268 & $<.0001$ & $\hat{0.0001}$ & 0.0294 & $\hat{0.0001}$ & 0.0009 \\
\hline $\begin{array}{l}\text { Current marijuana } \\
\text { use }\end{array}$ & $\begin{array}{l}582 \\
(33.8)\end{array}$ & $\begin{array}{l}217 \\
(26.5)\end{array}$ & $\begin{array}{l}126 \\
(35.5)\end{array}$ & $\begin{array}{l}110 \\
(35.5)\end{array}$ & $\begin{array}{l}129 \\
(54.0)\end{array}$ & 0.0018 & 0.0030 & ¿.0001 & 0.9981 & $\dot{0} 0001$ & $\overleftarrow{0} .0001$ \\
\hline $\begin{array}{l}\text { Ever synthetic } \\
\text { marijuana use }\end{array}$ & $\begin{array}{l}212 \\
(12.5)\end{array}$ & $\begin{array}{l}55( \\
6.8)\end{array}$ & $\begin{array}{l}40 \\
(11.7)\end{array}$ & $\begin{array}{l}42 \\
(13.6)\end{array}$ & $\begin{array}{l}75 \\
(32.3)\end{array}$ & 0.0060 & 0.0003 & $\begin{array}{l}< \\
0.0001\end{array}$ & 0.4665 & $\dot{0} 0001$ & $\hat{0} .0001$ \\
\hline $\begin{array}{l}\text { Ever prescription } \\
\text { pain medicine use }\end{array}$ & $\begin{array}{l}511 \\
(29.5)\end{array}$ & $\begin{array}{l}164 \\
(20.0)\end{array}$ & $\begin{array}{l}123 \\
(34.3)\end{array}$ & $\begin{array}{l}103 \\
(33.1)\end{array}$ & $\begin{array}{l}121 \\
(50.2)\end{array}$ & $\begin{array}{l}<.0001 \\
0.01\end{array}$ & $<.0001$ & $\begin{array}{l}<.0001 \\
0 .\end{array}$ & 0.7550 & 0.0001 & 0.0001 \\
\hline Ever cocaine use & $\begin{array}{l}132( \\
7.7)\end{array}$ & $\begin{array}{l}28( \\
3.4)\end{array}$ & $\begin{array}{l}24( \\
6.9)\end{array}$ & $\begin{array}{l}24( \\
7.7)\end{array}$ & $\begin{array}{l}56 \\
(24.7)\end{array}$ & 0.0090 & 0.0021 & $\begin{array}{l}<.0001 \\
0 .\end{array}$ & 0.6707 & $<.0001$ & $\overleftarrow{0.0001}$ \\
\hline Ever inhalant use & $\begin{array}{l}244 \\
(14.4)\end{array}$ & $\begin{array}{l}65( \\
8.1)\end{array}$ & $\begin{array}{l}61 \\
(17.4)\end{array}$ & $\begin{array}{l}42 \\
(13.9)\end{array}$ & $\begin{array}{l}76 \\
(32.3)\end{array}$ & $\hat{0} .0001$ & 0.0035 & $\begin{array}{l}< \\
0.0001\end{array}$ & 0.2190 & $\begin{array}{l}<.0001 \\
0 .\end{array}$ & $\stackrel{<}{0.0001}$ \\
\hline Ever heroin use & $\begin{array}{l}52( \\
3.0)\end{array}$ & $\begin{array}{l}7( \\
0.9)\end{array}$ & $\begin{array}{l}7( \\
2.0)\end{array}$ & $\begin{array}{l}6( \\
1.9)\end{array}$ & $\begin{array}{l}32 \\
(13.6)\end{array}$ & 0.0946 & 0.1367 & $\dot{0} 0001$ & 0.9137 & $\hat{0} .0001$ & $\hat{0} .0001$ \\
\hline $\begin{array}{l}\text { Ever } \\
\text { methamphetamine } \\
\text { use }\end{array}$ & $\begin{array}{l}74( \\
4.4)\end{array}$ & $\begin{array}{l}10( \\
1.2)\end{array}$ & $\begin{array}{l}15( \\
4.4)\end{array}$ & $\begin{array}{l}9( \\
2.9)\end{array}$ & $\begin{array}{l}40 \\
(17.1)\end{array}$ & 0.0008 & 0.0527 & $\hat{0} .0001$ & 0.3153 & $\hat{0} .0001$ & $\overleftarrow{0.0001}$ \\
\hline Ever ecstasy use & $\begin{array}{l}\text { 121 ( } \\
7.1)\end{array}$ & $\begin{array}{l}25( \\
3.1)\end{array}$ & $\begin{array}{l}17( \\
5.0)\end{array}$ & $\begin{array}{l}34 \\
(10.9)\end{array}$ & $\begin{array}{l}45 \\
(19.3)\end{array}$ & 0.1180 & $<.0001$ & ¿.0001 & 0.0046 & $\hat{0.0001}$ & 0.0060 \\
\hline Ever steroid use & $\begin{array}{l}53( \\
3.6)\end{array}$ & $\begin{array}{l}5( \\
0.7)\end{array}$ & $\begin{array}{l}14( \\
4.7)\end{array}$ & $\begin{array}{l}5( \\
1.8)\end{array}$ & $\begin{array}{l}29 \\
(15.6)\end{array}$ & $<.0001$ & 0.1163 & $\begin{array}{l}<.0001 \\
0 .\end{array}$ & 0.0516 & $\begin{array}{l}<.0001 \\
0.001\end{array}$ & $\hat{0} .0001$ \\
\hline $\begin{array}{l}\text { Illegal injected } \\
\text { drug use }\end{array}$ & $\begin{array}{l}39( \\
2.6)\end{array}$ & $\begin{array}{l}4( \\
0.6)\end{array}$ & $\begin{array}{l}8( \\
2.7)\end{array}$ & $\begin{array}{l}4( \\
1.4)\end{array}$ & $\begin{array}{l}23 \\
(12.4)\end{array}$ & 0.0039 & 0.1628 & $\begin{array}{l}< \\
0.0001\end{array}$ & 0.2846 & $\begin{array}{l}<.0001 \\
0.01\end{array}$ & $\hat{0} .0001$ \\
\hline $\begin{array}{l}\text { Ever sexual } \\
\text { intercourse }\end{array}$ & $\begin{array}{l}719 \\
(49.4)\end{array}$ & $\begin{array}{l}248 \\
(35.7)\end{array}$ & $\begin{array}{l}182 \\
(62.3)\end{array}$ & $\begin{array}{l}135 \\
(49.6)\end{array}$ & $\begin{array}{l}154 \\
(79.0)\end{array}$ & $\hat{0} .0001$ & 0.0001 & ¿.0001 & 0.0024 & 0.0001 & $\hat{0} 0.0001$ \\
\hline $\begin{array}{l}\text { Sex before } 13 \\
\text { years }\end{array}$ & $\begin{array}{l}101( \\
6.3)\end{array}$ & $\begin{array}{l}14( \\
1.8)\end{array}$ & $\begin{array}{l}28( \\
8.6)\end{array}$ & $\begin{array}{l}13( \\
4.5)\end{array}$ & $\begin{array}{l}46 \\
(20.7)\end{array}$ & $\dot{0.0001}$ & 0.0143 & ¿.0001 & 0.0391 & $\hat{0} .0001$ & $\hat{0} .0001$ \\
\hline $\begin{array}{l}\text { Multiple sex } \\
\text { partners }\end{array}$ & $\begin{array}{l}202 \\
(12.5)\end{array}$ & $\begin{array}{l}35( \\
4.5)\end{array}$ & $\begin{array}{l}66 \\
(20.1)\end{array}$ & $\begin{array}{l}34 \\
(11.7)\end{array}$ & $\begin{array}{l}67 \\
(29.9)\end{array}$ & $\hat{0.0001}$ & <. 0001 & <..0001 & 0.0044 & 0.0083 & $\begin{array}{l}<.0001 \\
0.01\end{array}$ \\
\hline $\begin{array}{l}\text { Current sexual } \\
\text { activity, } \geq 1 \text { people }\end{array}$ & $\begin{array}{l}574 \\
(35.5)\end{array}$ & $\begin{array}{l}207 \\
(26.8)\end{array}$ & $\begin{array}{l}133 \\
(40.5)\end{array}$ & $\begin{array}{l}102 \\
(35.2)\end{array}$ & $\begin{array}{l}132 \\
(58.9)\end{array}$ & $\hat{0.0001}$ & 0.0073 & <..0001 & 0.1694 & $\hat{0.0001}$ & $\hat{0.0001}$ \\
\hline $\begin{array}{l}\text { Alcohol/drugs and } \\
\text { sex }\end{array}$ & $\begin{array}{l}196 \\
(12.1)\end{array}$ & $\begin{array}{l}49( \\
6.3)\end{array}$ & $\begin{array}{l}47 \\
(14.3)\end{array}$ & $\begin{array}{l}31 \\
(10.5)\end{array}$ & $\begin{array}{l}69 \\
(31.5)\end{array}$ & $\hat{0.0001}$ & 0.0198 & $\hat{0.0001}$ & 0.1589 & $\langle .0001$ & $\hat{0.0001}$ \\
\hline Condom use, no & $\begin{array}{l}407 \\
(25.4)\end{array}$ & $\begin{array}{l}118 \\
(15.4)\end{array}$ & $\begin{array}{l}122 \\
(37.4)\end{array}$ & $\begin{array}{l}67 \\
(23.0)\end{array}$ & $\begin{array}{l}100 \\
(45.5)\end{array}$ & $\dot{0.0001}$ & 0.0035 & ¿.0001 & 0.0001 & 0.0609 & $\hat{0} .0001$ \\
\hline \multicolumn{12}{|c|}{$\begin{array}{l}\text { a. Risk factors that significantly associated with past- year suicide attempt. The full description for the question and response } \\
\text { classification were presented in Supplementary Table } 1 .\end{array}$} \\
\hline & & & & & & & & & & & \\
\hline
\end{tabular}




\begin{tabular}{|c|c|c|c|c|c|c|c|c|c|c|c|}
\hline \multirow[t]{2}{*}{ Risk behavior a } & \multirow[t]{2}{*}{ All } & \multicolumn{4}{|c|}{$\begin{array}{l}\text { Physical fight/ sexual violence } \\
\text { groups }\end{array}$} & \multicolumn{6}{|c|}{$P$ values for pair-wise comparisons ${ }^{b}$} \\
\hline & & $\begin{array}{l}-/- \\
\text { (Group } \\
1)\end{array}$ & $\begin{array}{l}-/+ \\
\text { (Group } \\
2 \text { ) }\end{array}$ & $\begin{array}{l}+/- \\
\text { (Group } \\
3 \text { ) }\end{array}$ & $\begin{array}{l}+/+ \\
\text { (Group } \\
4 \text { ) }\end{array}$ & $\begin{array}{l}\text { Group } \\
1 \text { vs } 2\end{array}$ & $\begin{array}{l}\text { Group } \\
1 \text { vs } 3\end{array}$ & $\begin{array}{l}\text { Group } \\
1 \text { vs } 4\end{array}$ & $\begin{array}{l}\text { Group } \\
2 \text { vs } 3\end{array}$ & $\begin{array}{l}\text { Group } \\
2 \text { vs } 4\end{array}$ & $\begin{array}{l}\text { Group } \\
3 \text { vs } 4\end{array}$ \\
\hline $\begin{array}{l}\text { Birth control pill } \\
\text { use, no }\end{array}$ & $\begin{array}{l}147( \\
9.3)\end{array}$ & $\begin{array}{l}48( \\
6.3)\end{array}$ & $\begin{array}{l}46 \\
(14.3)\end{array}$ & $\begin{array}{l}15( \\
5.3)\end{array}$ & $\begin{array}{l}38 \\
(17.8)\end{array}$ & $\hat{0} .0001$ & 0.5213 & $\hat{L}_{0.0001}$ & 0.0002 & 0.2790 & $\hat{0} .0001$ \\
\hline $\begin{array}{l}\text { Breakfast eating, } 0 \\
\text { days }\end{array}$ & $\begin{array}{l}349 \\
(23.3)\end{array}$ & $\begin{array}{l}152 \\
(21.0)\end{array}$ & $\begin{array}{l}75 \\
(25.2)\end{array}$ & $\begin{array}{l}65 \\
(23.1)\end{array}$ & $\begin{array}{l}57 \\
(29.2)\end{array}$ & 0.1477 & 0.4663 & 0.0153 & 0.5674 & 0.3191 & 0.1339 \\
\hline \multicolumn{12}{|l|}{$\begin{array}{l}\text { Cumulative } \\
\text { number of risk } \\
\text { factors }\end{array}$} \\
\hline Median (Q1, Q3) & $\begin{array}{l}6(3 \\
11)\end{array}$ & $\begin{array}{l}4(2, \\
7)\end{array}$ & $\begin{array}{l}8(5 \\
11)\end{array}$ & $\begin{array}{l}7(4, \\
11)\end{array}$ & $\begin{array}{l}13(8, \\
17)\end{array}$ & $\hat{0.0001}$ & $\hat{0.0001}$ & $\hat{0.0001}$ & 0.2800 & $\hat{0.0001}$ & $\hat{0} .0001$ \\
\hline Min, $\max$ & 0,40 & 0,22 & 0,36 & 0,26 & 0,40 & & & & & & \\
\hline \multicolumn{12}{|c|}{$\begin{array}{l}\text { a. Risk factors that significantly associated with past- year suicide attempt. The full description for the question and response } \\
\text { classification were presented in Supplementary Table } 1 .\end{array}$} \\
\hline
\end{tabular}

\section{Discussion}

The concurrence of physical fight and sexual violence substantially increased the relative risks for attempted suicide and injurious suicide attempt among adolescent suicide ideators. The pattern of associations were different among two sexes: among female, the association between physical fight and/or sexual violence and suicide behaviors were more prevalent; in males, the concurrence of physical fight and sexual violence was associated with remarkably higher risk for injurious suicide attempt. High rates of suicide-attempt associated risk behaviors, as well as a high cumulative number of risk behaviors were observed among the population with concurrent physical fight and sexual violence, especially among males.

Both physical fight and sexual violence had been known as risk factors/predictors for suicidal behaviors among adolescents and adults ${ }^{12-}$ 15. For example, the YRBS data showed that high school students who reported attempting suicide during the preceding 12 months were more likely also to have reported fighting than those who reported not attempting suicide $(61.5 \% \text { vs } 30.3 \%)^{15}$. Stack et al. reported that involvement in physical fighting raised the odds of a suicide attempt by 2.18 times $^{13}$. A experience of sexual violence was found to be associated with increased risk of suicide attempt among both high school male and female students $(\mathrm{OR}=11.3$ and 4.54 , respectively; $P<$ $0.05)^{14}$. Consistent with previous findings, our analyses showed both factors were associated with increased risk of suicide attempt. The potential role of physical fight and sexual violence in the suicidal behavior trajectory may be understood within the context of the interpersonal theory of suicide ${ }^{2}$. According to the interpersonal theory, three constructs, namely thwarted belongingness, perceived burdensomeness, and the acquired capability for suicide, are needed for an individual to attempt suicide. The capability for suicide could be acquired by both increased physical pain tolerance and reduced fear of death through habituation to the physically painful and fearful aspects of self-harm ${ }^{2}$. Drawing on the interpersonal theory of suicide, physical fights may serve as a risk factor for suicidal behaviors by increasing the individual's acquired capability for suicide. In support of this explanation, one study had reported that individuals who reported engaging in physical fights had higher acquired capability scores ${ }^{21}$. The experience of sexual violence might lead to suicidal desires by increasing the victim's sense of perceived burdensomeness and thwarted belongingness; moreover, it may increase the victim's capability for suicide by increasing the habituation to the pain and fear in suicide ${ }^{14}$.

Our analyses showed that among the four study groups (physical fight - / sexual violence -, physical fight - / sexual violence + , physical fight + / sexual violence - , and physical fight + / sexual violence + ), the group with concurrent physical fight and sexual violence had the highest risks of suicide attempt $(R R=2.01,95 \% \mathrm{Cl}: 1.74,2.31)$, suicide plan $(R R=1.22,95 \% \mathrm{Cl}: 1.11,1.33)$, and injurious suicide attempt (RR $=4.03,95 \% \mathrm{Cl}: 2.81,5.78)$. Since physical fight and sexual violence along could increase the individual's capability for suicide, it is possible that the concurrence of two exposures could further increased the individual's capability for suicide, resulting in higher rates of suicide attempt and suicide plan. More importantly, the concurrence of physical fight and sexual violence raised the risk of injurious suicide attempt by 4 times, suggesting that the combination of physical fight and sexual violence is associated with more severe forms of suicide attempt. This finding has both theoretical and practical implications. First, it suggests that when conceptualize theory of suicide, it is 
necessary to consider how multiple risk factors could influence the suicide behavior. The acquired capability for suicide might accumulated through multiple risk factors, and the amplified capability could result in more lethal suicide behaviors. For practical uses, our findings demonstrated that in searing for predicting factors for suicide attempt among ideators, it is imperative to examine the effects of different combinations of risk factors. And our results emphasis the need for targeted prevention and intervention efforts in youth with concurrent experience of physical fight and sexual violence.

Our study observed associations between physical fight/sexual violence and a wide variety of health-risk behaviors, many of which had been reported in previous studies ${ }^{17,19,22,23}$. For example, weapon-carrying was found to be associated with increased involvement in physical fighting ${ }^{19}$, and early alcohol use initiation and heavy drinking was associated with comorbid fight and suicide attempts among adolescent ${ }^{23}$; a history of adolescent sexual victimization was found to be associated with increased likelihood of cigarette smoking, marijuana use, multiple sexual partners, and early sexual intercourse among college women ${ }^{17}$. Considering that the risk behavior we investigated were selected from factors that associated with the risk of suicide attempt, the observed association between physical fight and/or sexual violence and the risk of suicide attempt could be a result of combined effects of the clustered risk factors. The clustered factors could interact as both cause and effect, and influenced the suicidal behavior through complex pathways, therefore isolating the effects of single factors might not practical. Also, interventions targeting single factors might not powerful enough for the prevention of suicidal attempts. The problem behavior theory suggested that the risk-health behaviors might cluster together because they serve similar psychological and social developmental functions ${ }^{20}$. Therefore, for the purpose of developing intervention plans, it is important to identify the key factors that leads to subsequent risk behaviors and to identify reversible factors that could serve as intervention targets.

Among both sexes, the concurrency of physical fight and sexual violence was associated with a high risk for injurious suicide attempt. $26.5 \%$ of female students with concurrent physical fight and sexual violence reported injurious suicide attempt; among male students, the rate was $32.8 \%$. The $\mathrm{RR}$ for injurious suicide attempt was remarkably higher among male $(\mathrm{RR}=6.19,95 \% \mathrm{Cl}: 3.3,12.14)$ than female $(\mathrm{RR}=$ $3.25,95 \% \mathrm{Cl}: 2.09,5.04)$, using the risk in the (physical fight -/ sexual violence -) group in the same sex as the reference. Sex difference in the suicidal behavior has consistently been observed. In most part of the world, females are more likely than men to attempt suicide, and they are less likely to die by suicide ${ }^{16}$. One explanation from the interpersonal theory of suicide is that females are less likely to develop the acquired capability for suicidal behavior that are men, because females generally experiences fewer events that could reduce their fear of self-injury through habituation (e.g., exposure to guns, physical fights, violent sports, etc.), and females have lower pain and fear tolerance than men ${ }^{2}$. Our finding supported this explanation in that among the youth with concurrent physical fight and sexual violence, males reported more health-risk behaviors than females (male: median $=14$, range: $2-40$; female: median $=12$, range: $0-33$; $P=0.0023$ ). This finding adds empirical evidence for the understanding of sex difference in suicidal behaviors, and again strengths the importance of examining an individual's risk factor profile when evaluating the suicide risk.

Strengths of this study include the novel investigation of the association between concurrent physical fight and sexual violence and the risk of suicide attempt among adolescent suicide ideators, and the cumulative number of suicide risk factors among several sub-populations. In searching for risk factors that could differentiate attempters from ideators, most of previous studies examined the effects of single factors. The findings of our study emphases that in both researches and intervention practices, the individual's risk of suicide behaviors should be conceptualized as a combined effects of multiple factors. The cumulative number of suicide risk factors also helps understand the remarkable higher rates of injurious suicide attempt among adolescent males with concurrent physical fight and sexual violence. Besides, when estimating the strength of association between risk factors and suicide behaviors, unlike studies that used logistic regression to estimate the $\mathrm{OR}$, we used log binomial regression to estimate the RR and AR which are more intuitive and can be better interpreted ${ }^{24}$.

This study has several limitations. First, the cross-sectional design of the study limits our ability to make causal inferences. It is possible that some of the reported experiences of physical fight and sexual violence were occurred after the suicide attempt. Nevertheless, the identified patterns could still be informative for the understanding of how the suicide-related risk behaviors clustered and for the identification of high risk populations that need multiple behavior interventions. Longitudinal studies are needed to test the causality relationship between sexual violence, physical fight, and suicide behaviors. Second, the YRBS data are collected from self-reported questionnaires, the answers would subject to recall bias and classification error which was caused by individual's different understanding of the queries. Third, the YRBS is a school based survey, therefore may not representative for adolescents who did not attend school. Lastly, findings of this study are draw from the adolescents from the United States, the combined effects of could be different under different culture and social-development conditions ${ }^{25}$. To improve the understanding of the suicide behaviors and promote effective interventions, future research is needed to validate these findings in different counties and populations. 
To conclude, the concurrence of physical fight and sexual violence substantially increased the risks for attempted suicide and injurious suicide attempt among adolescent suicide ideators. A wide variety of risk behaviors clustered with the concurrence of physical fight and sexual violence, especially among males, which possibly lead to the higher rate of injurious suicide attempt among male students. Further study is needed to explore the mechanism of how combined risk factors works in the suicide pathways. And to guide the development of suicide intervention strategies, it is important to identify the key factors that leads to subsequent risk behaviors and to identify reversible factors that could serve as intervention targets.

\section{Declarations}

\section{Author contributions}

X.L. contributed to the conception of the work. X.L., S.X. and J.D. analyzed the data and wrote the main manuscript text. All authors reviewed the manuscript.

\section{Additional Information}

\section{Competing interests}

The authors declare no competing interests.

\section{Data availability}

The datasets generated during and/or analysed during the current study are available at: https://www.cdc.gov/healthyyouth/data/yrbs/data.htm

\section{References}

1. WHO. Suicide worldwide in 2019: global health estimates (World Health Organization, Geneva, 2021).

2. Van Orden, K. A. et al. The interpersonal theory of suicide. Psychol Rev, 117, 575-600 https://doi.org/10.1037/a0018697 (2010).

3. Ivey-Stephenson, A. Z. et al. Suicidal Ideation and Behaviors Among High School Students - Youth Risk Behavior Survey, United States, 2019. MMWR supplements 69, 47-55, doi:10.15585/mmwr.su6901a6 (2020).

4. Fergusson, D. M., Horwood, L. J., Ridder, E. M. \& Beautrais, A. L. Suicidal behaviour in adolescence and subsequent mental health outcomes in young adulthood. Psychological medicine, 35, 983-993 https://doi.org/10.1017/s0033291704004167 (2005).

5. Reinherz, H. Z., Tanner, J. L., Berger, S. R., Beardslee, W. R. \& Fitzmaurice, G. M. Adolescent suicidal ideation as predictive of psychopathology, suicidal behavior, and compromised functioning at age 30. The American journal of psychiatry, 163, 1226-1232 https://doi.org/10.1176/appi.ajp.163.7.1226 (2006).

6. May, A. M. \& Victor, S. E. From ideation to action: recent advances in understanding suicide capability. Curr Opin Psychol, 22, 1-6 https://doi.org/10.1016/j.copsyc.2017.07.007 (2018).

7. Steele, M. M. \& Doey, T. Suicidal behaviour in children and adolescents. part 1: etiology and risk factors. Canadian journal of psychiatry. Revue canadienne de psychiatrie, 52, 21-33 (2007).

8. Wan, G. W. \& Leung, P. W. Factors accounting for youth suicide attempt in Hong Kong: a model building. J Adolesc, 33, 575-582 https://doi.org/10.1016/j.adolescence.2009.12.007 (2010).

9. Ribeiro, J. D. et al. Self-injurious thoughts and behaviors as risk factors for future suicide ideation, attempts, and death: a metaanalysis of longitudinal studies. Psychological medicine, 46, 225-236 https://doi.org/10.1017/s0033291715001804 (2016).

10. Franklin, J. C. et al. Risk factors for suicidal thoughts and behaviors: A meta-analysis of 50 years of research. Psychological bulletin, 143, 187-232 https://doi.org/10.1037/bul0000084 (2017).

11. May, A. M., Czyz, E. K. \& West, B. T. Differentiating Adolescent Suicide Attempters and Ideators: A Classification Tree Analysis of Risk Behaviors. The Journal of adolescent health: official publication of the Society for Adolescent Medicine, 67, 837-850 https://doi.org/10.1016/j.jadohealth.2020.04.018 (2020).

12. Juan, W., Xiao-Juan, D., Jia-Ji, W., Xin-Wang, W. \& Liang, X. The associations between health risk behaviors and suicidal ideation and attempts in an urban Chinese sample of adolescents. Journal of affective disorders, 126, 180-187 https://doi.org/10.1016/j.jad.2010.02.121 (2010). 
13. Stack, S. Differentiating suicide ideators from attempters: violence-a research note. Suicide Life Threat Behav, 44, 46-57 https://doi.org/10.1111/sltb.12054 (2014).

14. Baiden, P. et al. Sex differences in the association between sexual violence victimization and suicidal behaviors among adolescents. Journal of Affective Disorders Reports, 1, 100011 https://doi.org/10.1016/j.jadr.2020.100011 (2020).

15. CDC. Suicide attempts and physical fighting among high school students-United States, 2001. MMWR. Morbidity and mortality weekly report, 53, 474-476 (2004).

16. Beautrais, A. L. Gender issues in youth suicidal behaviour. Emergency medicine (Fremantle, W.A.) 14, 35-42, doi:10.1046/j.14422026.2002.00283.x (2002).

17. Gidycz, C. A., Orchowski, L. M., King, C. R. \& Rich, C. L. Sexual victimization and health-risk behaviors: a prospective analysis of college women. Journal of interpersonal violence, 23, 744-763 https://doi.org/10.1177/0886260507313944 (2008).

18. Lowry, R., Crosby, A. E., Brener, N. D. \& Kann, L. Suicidal thoughts and attempts among u.s. High school students: trends and associated health-risk behaviors, 1991-2011. The Journal of adolescent health: official publication of the Society for Adolescent Medicine, 54, 100-108 https://doi.org/10.1016/j.jadohealth.2013.07.024 (2014).

19. Lowry, R., Powell, K. E., Kann, L., Collins, J. L. \& Kolbe, L. J. Weapon-carrying, physical fighting, and fight-related injury among U.S. adolescents. American journal of preventive medicine, 14, 122-129 https://doi.org/10.1016/s0749-3797(97)00020-2 (1998).

20. Jessor, R. Problem-behavior theory, psychosocial development, and adolescent problem drinking. British journal of addiction, 82, 331342 https://doi.org/10.1111/j.1360-0443.1987.tb01490.x (1987).

21. Van Orden, K. A., Witte, T. K., Gordon, K. H., Bender, T. W. \& Joiner, T. E. Jr. Suicidal desire and the capability for suicide: tests of the interpersonal-psychological theory of suicidal behavior among adults. Journal of consulting and clinical psychology, 76, 72-83 https://doi.org/10.1037/0022-006x.76.1.72 (2008).

22. Basile, K. C., Clayton, H. B., Rostad, W. L. \& Leemis, R. W. Sexual Violence Victimization of Youth and Health Risk Behaviors. American journal of preventive medicine, 58, 570-579 https://doi.org/10.1016/j.amepre.2019.11.020 (2020).

23. Swahn, M. H., Bossarte, R. M., Palmier, J. B. \& Yao, H. Co-Occurring Physical Fighting and Suicide Attempts among U.S. High School Students: Examining Patterns of Early Alcohol Use Initiation and Current Binge Drinking. West J Emerg Med, 14, 341-346 https://doi.org/10.5811/westjem.2013.3.15705 (2013).

24. Grant, R. L. Converting an odds ratio to a range of plausible relative risks for better communication of research findings. $B M J, 348$, f7450 https://doi.org/10.1136/bmj.f7450 (2014).

25. Harel-Fisch, Y., Abdeen, Z., Walsh, S. D., Radwan, Q. \& Fogel-Grinvald, H. Multiple risk behaviors and suicidal ideation and behavior among Israeli and Palestinian adolescents. Soc. Sci. Med, 75, 98-108 https://doi.org/10.1016/j.socscimed.2012.03.005 (2012).

26. Underwood, J. M. et al. Overview and Methods for the Youth Risk Behavior Surveillance System - United States, 2019. MMWR supplements 69, 1-10, doi:10.15585/mmwr.su6901a1 (2020).

\section{Figures}




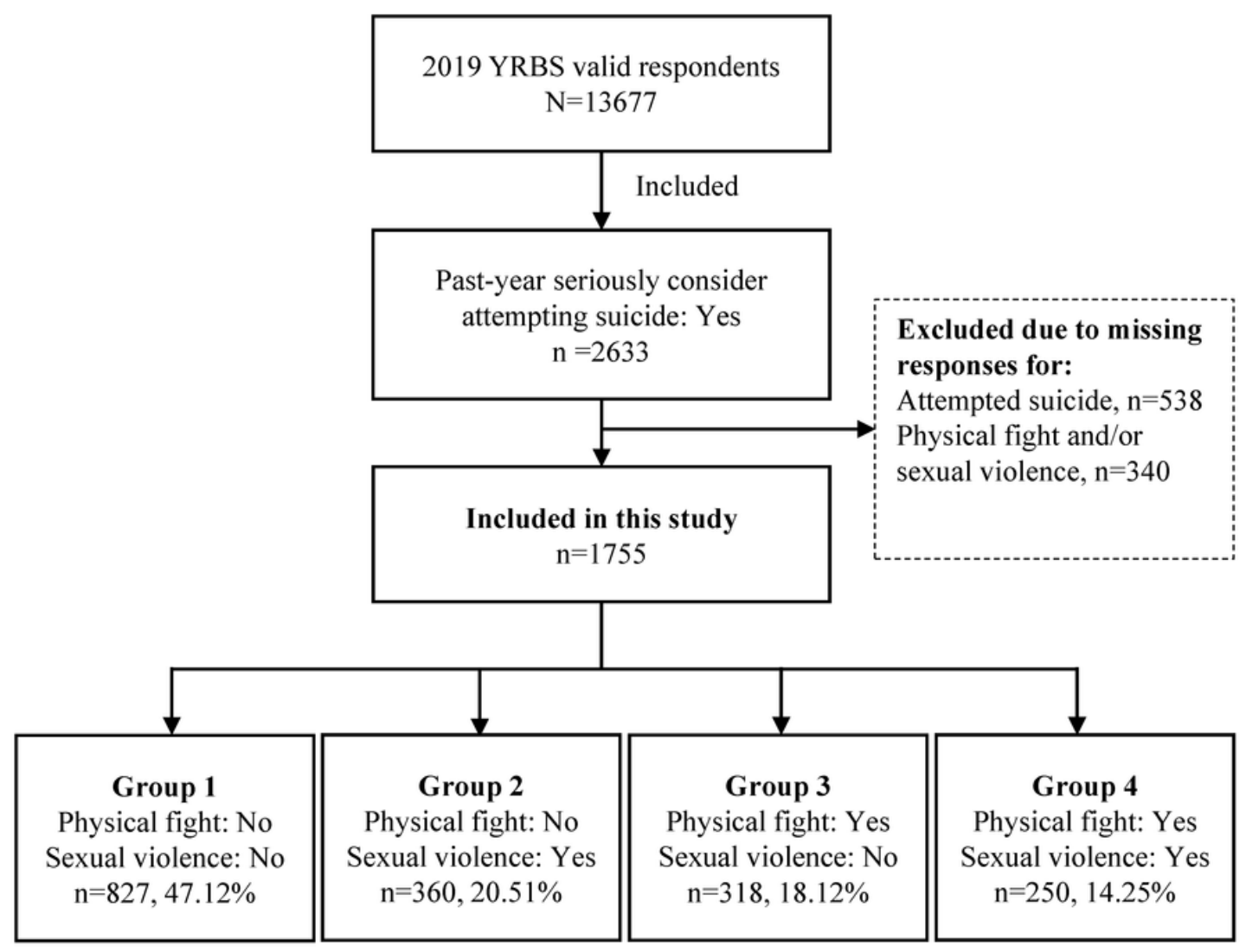

Figure 1

Flow diagram of study population 

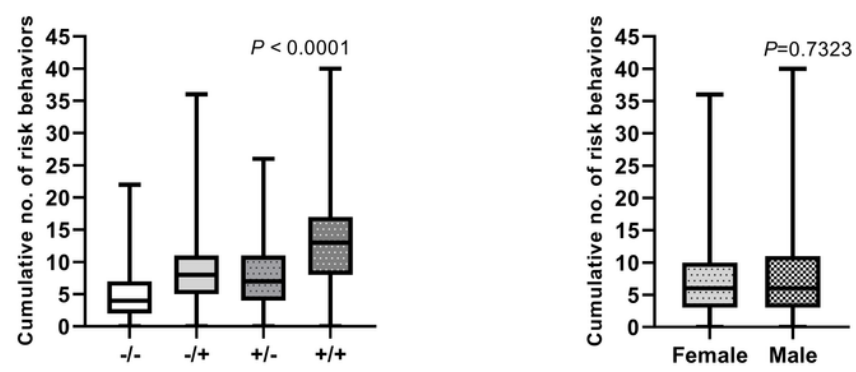

Physical fight/ sexual violence groups
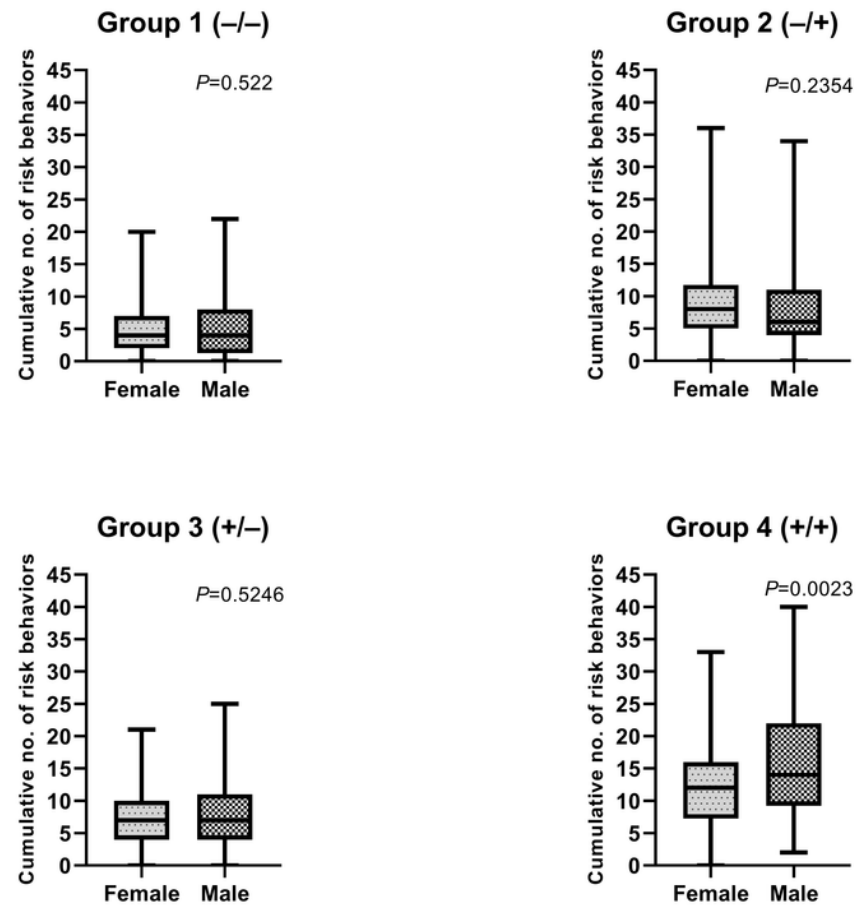

\section{Figure 2}

Cumulative number of risk behaviors among students who reported past-year seriously considered attempting suicide, according to physical fight/ sexual violence groups and sex

\section{Supplementary Files}

This is a list of supplementary files associated with this preprint. Click to download.

- Supplementarymaterial.pdf 